\title{
Making the transition in difficult airway management: Are new perspectives in Critical Emergency Medicine nurses curricula needed?
}

\author{
Ileana Lulic', Saqr AlHemeiri', AlAnood Bin Sulaiman1, Afaf Sayed Jaafer', Mirna Diab1, Mahmoud \\ Mustafa², Dinka Lulic ${ }^{3}$ \\ ${ }^{1}$ Training and Development Center, Ministry of Health and Prevention, Sharjah, United Arab Emirates \\ 2Sudan Resuscitation Council, Khartoum, Sudan \\ ${ }^{3}$ Department of Emergency Medicine, Clinical Hospital Center Zagreb, Zagreb, Croatia \\ E-mail address: ileanalulic@gmail.com
}

\section{Background}

Airway management is of paramount importance in Critical Emergency Medicine (CREM) setting. Unanticipated difficult airway (DA) represents a major challenge in high acuity CREM airway emergencies and may result in significant morbidity and mortality. Therefore, all CREM personnel must be familiar with well - established framework for unanticipated DA management. Subsequently, CREM concept of multidisciplinary team approach in managing DA highlighted nurse's role as pivotal. Updated Difficult Airway Society (DAS) 2015 Guidelines provide a strategy to manage airway crisis depending on individual experience, training and comfort of use.

In our study we aimed to investigate United Arab Emirates (UAE) Ministry of Health and Prevention (MOHAP) CREM nurses optimal practice during DA cases.

\section{Oـ} Training and Development Center

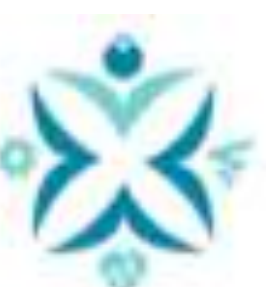

UNITEO ARAB EMILATES

MINISTRY OF HEALTH \& PREVENTION

\section{Conclusions}

Our results suggest CREM nurses have a strategy of utilising optimal emergency DA algorithms. However, we strongly recommend directing resources towards implementation and training of updated DAS 2015 Guidelines, providing a sequential series of plans to be used when dealing with DA, into CREM nurses curricula.

\section{Materials and Methods}

A total of 145 (56\%) female nurses, aged from 25 to 58 years (median 38 ), completed a survey prior to their enrolment into Airway Basics Course for Critical Emergency Medicine. The survey consisted of demographic data and 10 open questions regarding management of the emergent DA their encounter in daily practice.

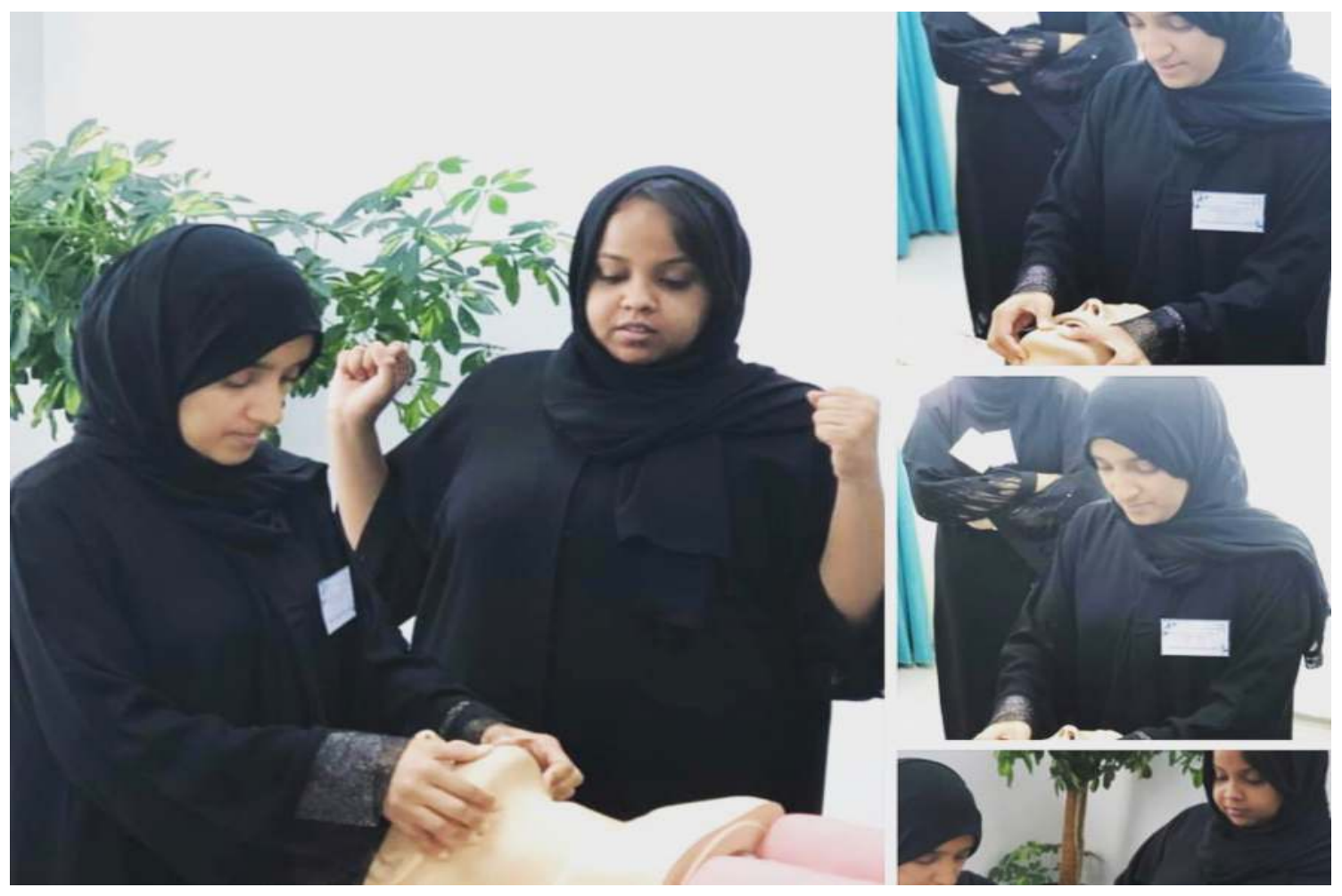

\section{Results}

The majority of nurses were from Emergency Department $(52 \%)$, followed by ones working in Intensive Care Units (48\%). More than two thirds $(88 \%)$ of nurses are familiar with DA algorithms instituted in their workplace. However, less than a third $(15 \%)$ of these institutional DA algorithms are in concordance with DAS 2015 Guidelines. 